УДК 504.064.36; 504.064.38

DOI: $10.15827 / 0236-235 X .136 .649-659$

Дата подачи статьи: 28.05.21, после доработки: 23.06.21

2021. T. 34. № 4. C. 649-659

\title{
Анализ состояния окружающей среды и вопросы нейтрализачии выбросов в Китае на примере Пекина
}

\author{
Р.И. Сольнииев 1, д.m.н., профрессор, remira70@mail.ru \\ Мэй Ван ${ }^{1}$, аспирант, 18361206765@163.com \\ C.A. Kузьмин ${ }^{1}$, к.m.н., KSA84@yandex.ru \\ Г.А. Куприянов 1,2, программист, научный сотрудник, science@help-in.ru \\ ${ }^{1}$ Санкт-Петербургский государственный электротехнический университет "АЭТИ" \\ им. В.И. Ульянова (Иенина), г. Санкт-Петербург,197376, Россия \\ ${ }^{2}$ Международный институт инжиниринга в экологии и безопасности человека, \\ г. Санкт-Петербург, 197376, Россия
}

Статья посвящена проблеме компенсации выбросов загрязняющих веществ в атмосферу. Применяется комплексная оценка концентрации выбросов в пространстве. Строится соответствующая модель распространения выбросов в зависимости от типа источника загрязнения. Анализ проводится на основе данных по выбросам загрязняющих веществ в конкретном районе Пекина (Китай). На основе анализа основных стационарных и подвижных (автотранспорта) источников загрязнения в этом районе определены градиенты концентрации выбросов в атмосферу и уровень качества воздуха в течение дня.

Предлагаются подходы и конструктивные решения по компенсации выбросов на основе концепции построения замкнутой системы «Природа-Техногеника». Такие замкнутые системы управления включают средства компенсации выбросов как стационарных, так и нестационарных источников загрязняющих веществ. Предлагается решение задач синтеза управлений по критерию минимизации выбросов в экстремальных сечениях потоков выбросов.

Построение замкнутых систем управления компенсацией выбросов связано с формированием соответствующей базы знаний, которая создается на основе составляющих онтологии, в том числе видов загрязняющих атмосферу веществ и способов их компенсации. В онтологии присутствуют такие понятия, как окружающая среда, в которой в данный момент находится человек, загрязняющее вещество в данный момент в среде и текущая степень компенсации источников загрязняющих веществ. В дополнение к онтологии в базе знаний присутствуют правила, которые на основании состояния окружающей среды (в том числе городской инфраструктуры или закрытых помещений) и вида загрязняющих веществ (пыль, токсичные или нейтральные газы) позволяют сделать вывод о степени эффективности компенсации предлагаемыми средствами. Онтология разрабатывалась в редакторе Protégé, а веб-приложение для работы с базой знаний с помощью технологии JSP и языка программирования Јava. На основе базы знаний строятся геоинформационные системы для визуализации данных и технологии блокчейн для регистрации и надежного хранения данных. В статье показана перспектива развития этого подхода для решения комплексной проблемы компенсации выбросов как от стационарных источников загрязнений, так и от автотранспорта.

Ключевые слова: выбросы, вероятностная характеристика, предельно допустимая концентрация, модель диффузии и конвекиии, нейтрализация загрязняющего вещества, система мониторинга, замкнутая система управления, база знаний, хранение данных, блокчейн, геоинформационныле системьн.

Обнародование политики национальной стратегии развития новой энергетики, такой как «План возрождения и развития новой энергетики» Национальной энергетической комиссии Китая, показало, что вопросы энергетики и окружающей среды поднялись до уровня национальной безопасности и требуются конструктивные подходы к сокращению выбросов загрязняющих вещзеств (ЗВ).

В статье на основе анализа ситуации с выбросами в Пекине рассматриваются математические модели оценки вероятностных ха- рактеристик разных источников $3 B$ (ИЗВ). Предлагается применение замкнутой системы управления нейтрализацией выбросов (ЗСУНВ) как для стационарных, так и для нестационарных (автотранспорта) ИЗВ.

\section{Анализ качества воздуха на основе комплексной оценки 3В}

Анализ качества воздуха основан на оценке комплексного индекса - аналога предельно допустимой концентрации (ПДК) [1]. 
Показатели качества воздуха (IAQI) [2] и соответствующие им ПДК представлены в таблице 1 . В таблице отражены среднесуточные показатели концентрации 3В, за исключением показателя $\mathrm{O}_{3}$, представляющего собой среднюю концентрацию за час.

По величинам ПДК, приведенным в таблице, можно рассчитать комплексную оценку качества воздуха по конкретному ИЗВ $P$. Расчет такой оценки [3] по загрязняющему элементу $P$ осуществляется по формуле

$$
I A Q I_{P}=\frac{I A Q I_{H i}-I A Q I_{L o}}{B P_{H i}-B P_{L o}}\left(C_{P}-B P_{L o}\right)+I A Q I_{L o},
$$

где $I A Q I$ - рейтинг качества воздуха; $P$ - загрязняющий элемент (ИЗВ); $I A Q I_{p}$ - оценка качества воздуха по загрязняющему элементу $P ; C_{p}$ - значение концентрации ЗВ от ИЗВ $P$; $B P_{H i}$ - максимальное значение предельной концентрации $3 \mathrm{~B} C_{p}$ из таблицы $1 ; B P_{L o}-$ минимальное значение концентрации $C_{p}$; $I A Q I_{H i}$ - оценка качества воздуха, соответствующая $B P_{H i}$; $I A Q I_{L o}$ - оценка качества воздуха, соответствующая $B P_{L o}$.

Приведенный расчет характеристики точечного ИЗВ служит для получения начальных и граничных условий в уравнении диффузии и конвекции [4]. Учет влияния ветра на перенос факела проводится путем расчета скорости ветра на выходе трубы.
Скорость ветра на разных высотах по отношению к скорости ветра на выходе трубы рассчитывается следующим образом [5]:

$$
u= \begin{cases}u_{1}\left(z_{2} / z_{1}\right)^{m}, & z_{2} \leq 200 \mathrm{M}, \\ u_{1}\left(200 / z_{1}\right)^{m}, & z_{2}>200 \mathrm{M},\end{cases}
$$

где $z_{1}$ - высота $10 \mathrm{м} ; z_{2}$ - высота от земли; $u_{1}$ средняя скорость ветра на высоте $z_{1} ; m$ - индекс профиля ветра.

Значения $m$ показаны в таблице 2 .

\section{Анализ основных источников ЗВ в Пекине}

В БД мониторинга в Пекине [3] можно обнаружить, что из-за различий в промышленной структуре города основные ИЗВ в разных районах не совпадают. Основные ИЗВ и их параметры показаны на рисунке 1 (единица измерения - 10000 тонн).

Как видно из рисунка, основными в Пекине являются стационарные (отопление и промышленные предприятия), применяющие в качестве топлива уголь, и нестационарные (автотранспорт на углеводородном топливе) ИЗВ. Интенсивность ИЗВ [6] - исходный параметр для его оценки.

Интенсивность такого ИЗВ, как автотранспорт, - это выброс ЗВ в единицу вре-

Таблииа 1

ПДК ЗВ, соответствующая различным уровням индексов качества воздуха в Китае и международным (мг/м³

Table 1

The maximum permissible concentration of pollutant items corresponding to various Chinese and International levels of air quality indexes $\left(\mu \mathrm{g} / \mathrm{Nm}^{3}\right)$

\begin{tabular}{|c|c|c|c|c|c|c|c|c|c|c|c|c|}
\hline \multirow{2}{*}{ IAQI } & \multicolumn{2}{|c|}{$\mathrm{SO}_{2}$} & \multicolumn{2}{c|}{$\mathrm{NO}_{2}$} & \multicolumn{2}{c|}{ PM10 } & \multicolumn{2}{c|}{ PM2.5 } & \multicolumn{2}{c|}{ CO } & \multicolumn{2}{c|}{$\mathrm{O}_{3}$} \\
\cline { 2 - 15 } & CHN & INT & CHN & INT & CHN & INT & CHN & INT & CHN & INT & CHN & INT \\
\hline 50 & 0.05 & 0.078 & 0.04 & 0.04 & 0.05 & 0.05 & 0.035 & 0.015 & 0.002 & 0.0045 & 0.16 & 0.126 \\
\hline 100 & 0.15 & 0.37 & 0.08 & 0.08 & 0.15 & 0.15 & 0.075 & 0.04 & 0.004 & 0.01 & 0.2 & 0.16 \\
\hline 150 & 0.48 & 0.58 & 0.18 & 0.18 & 0.25 & 0.25 & 0.12 & 0.065 & 0.014 & 0.014 & 0.3 & 0.2 \\
\hline 200 & 0.8 & 0.79 & 0.28 & 0.28 & 0.35 & 0.35 & 0.15 & 0.15 & 0.024 & 0.017 & 0.4 & 0.25 \\
\hline 300 & 1.6 & 1.57 & 0.57 & 0.57 & 0.42 & 0.42 & 0.25 & 0.25 & 0.036 & 0.034 & 0.8 & 0.86 \\
\hline 400 & 2.1 & - & 0.75 & - & 0.5 & - & 0.35 & - & 0.048 & - & 1 & - \\
\hline 500 & 2.62 & 2.62 & 0.94 & 0.94 & 0.6 & 0.6 & 0.5 & 0.5 & 0.06 & 0.057 & 1.2 & 1.28 \\
\hline
\end{tabular}

Таблий 2

Значение индекса профиля ветра (m) при различных условиях стабильности

Table 2

The value of wind profile index $(m)$ under various stability conditions

\begin{tabular}{|l|c|c|c|c|c|c|}
\hline \multirow{2}{*}{ Местность } & \multicolumn{7}{|c|}{ Степень устойчивости } \\
\cline { 2 - 7 } & $\mathrm{A}$ & $\mathrm{B}$ & $\mathrm{C}$ & $\mathrm{D}$ & $\mathrm{E}$ & $\mathrm{F}$ \\
\hline Город & 0.10 & 0.15 & 0.20 & 0.25 & 0.30 & 0.30 \\
\hline Пригород & 0.07 & 0.07 & 0.10 & 0.15 & 0.25 & 0.25 \\
\hline
\end{tabular}




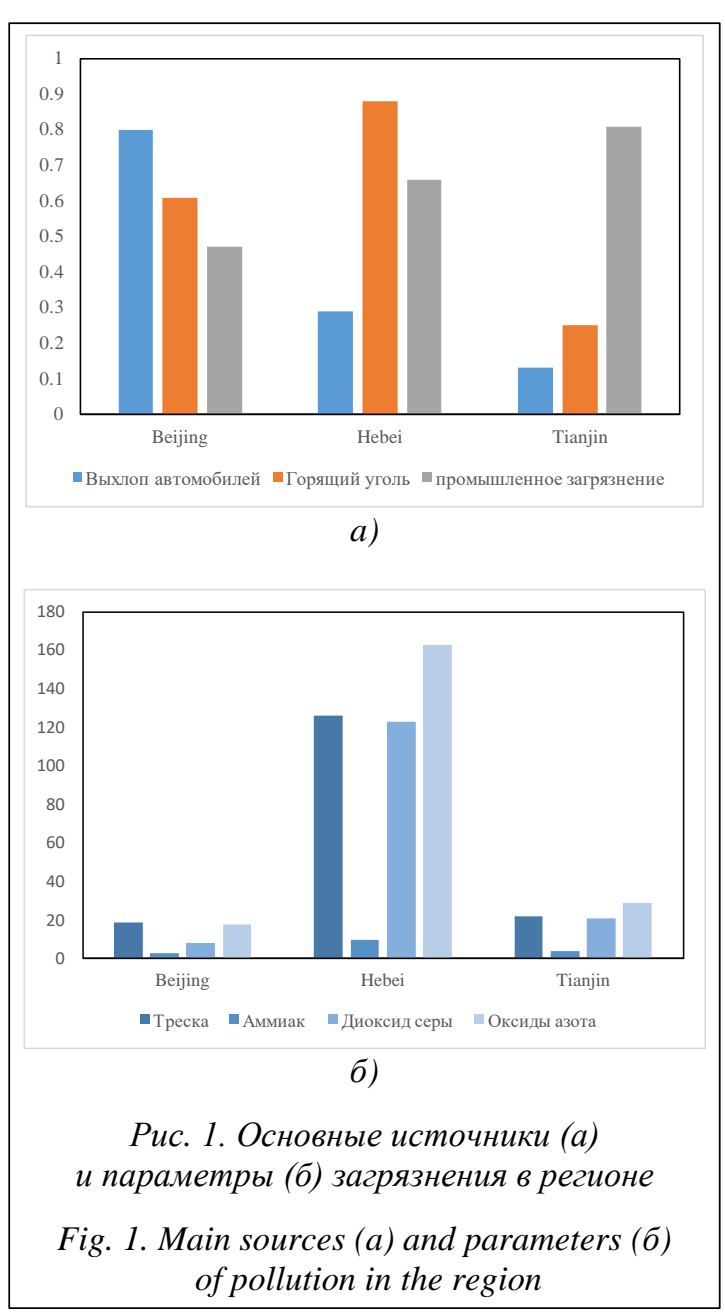

мени (мг/ч). Главным источником загрязнения являются выхлопные газы транспортных средств, которые в основном включают оксиды углеводорода и азота. Так, по приведенным в [7] данным, интенсивность источников выбросов оксидов азота определяется по следующей формуле:

$$
Q_{\mathrm{NO} x}=1.63 B_{1}\left(N_{1} \beta_{1}+0.000938\right),
$$

где $B_{1}-$ количество израсходованного топлива; $N_{1}$ - содержание азота в топливе (обычно 0,85 ); $\beta_{1}$ - степень конверсии азота в топливе.

Для стационарных ИЗВ источником загрязнения является сжигание угля, вследствие чего образуется диоксид серы - $\mathrm{SO}_{2}$. Формула расчета интенсивности источника $\mathrm{SO}_{2}$ следующая: $Q_{\mathrm{so}_{2}}=0.8 \times 2 B_{2} N_{2}\left(1-\beta_{2}\right)$, где $B_{2}$ - количество сожженного угля; $N_{2}$ - содержание серы в топливе; $\beta_{2}-$ эффективность компенсации.

Интенсивность ИЗВ, выделяющего дым и пыль, определяется по формуле

$$
Q_{\text {пыль }}=B_{3} N_{3} L\left(1-\beta_{3}\right) \text {, }
$$

где $B_{3}$ - количество израсходованного угля; $N_{3}$ - количество угольной пыли; $\beta_{3}-$ эффективность удаления пыли.

На рисунке 2 показан тренд изменения концентрации трех вышеупомянутых основных загрязнителей.

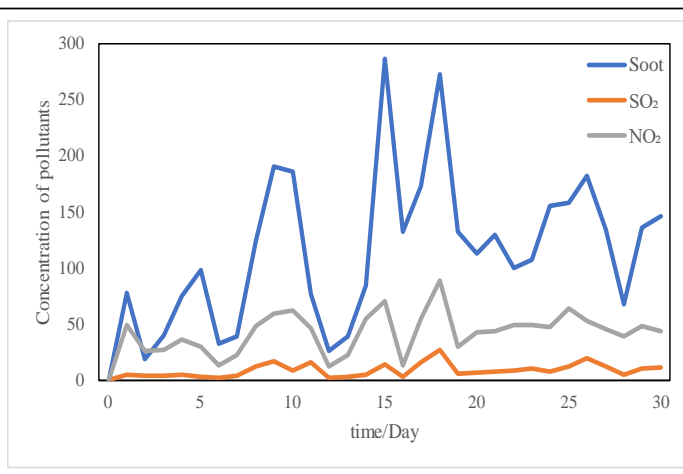

Рис. 2. Изменение концеентрации трех $3 B$

Fig. 2. The changes in concentrations of three pollutants

При обработке результатов мониторинга в Пекине [8] принималось, что транспортные средства являются линейными ИЗВ, а стационарные источники - точечными.

В качестве примера расчета изменения концентрации 3В только от автотранспорта покажем изменение концентрации загрязнения и оценку качества воздуха на второй, четвертой и шестой кольцевых дорогах в Пекине в 8.00, 12.00 и 21.00 в определенный день. Выбросы выхлопных газов автомобильного транспортного потока можно приближенно рассматривать как непрерывный бесконечный линейный источник. Интенсивность источника загрязнения рассчитывается по формуле $Q=\Sigma Q_{i}=\Sigma E F_{m} q_{m} B$, где $Q_{i}$ - сильный источник выбросов транспортного средства на $i$-й дороге; $E F_{m}-$ коэффициент выбросов автомобилей $m$-типа; $q_{m}$ - расход автомашины $m$-типа; $B$ - коэффициент преобразования единицы, принимаемый равным 1/3 600 .

Транспортный поток является одним из важных рабочих параметров [9]. Время максимального объема трафика - с 8:00 до 18:00, включая утренние и вечерние часы пик, а также полдень, когда объем трафика относительно невелик [10]. Следовательно, более подходящим является непрерывное наблюдение с 8:00 до 18:00 в день с частотой в 1 час. Используя соответствующие данные и расчеты на MATLAB, можно получить графики изменения концентраций ЗВ (рис. 3). 


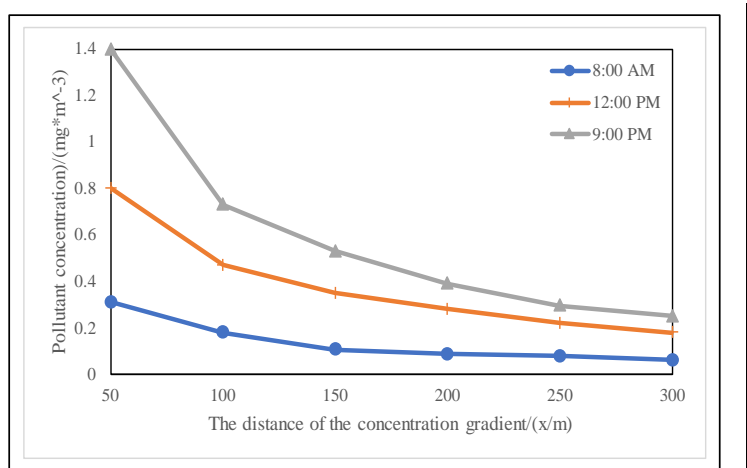

a)

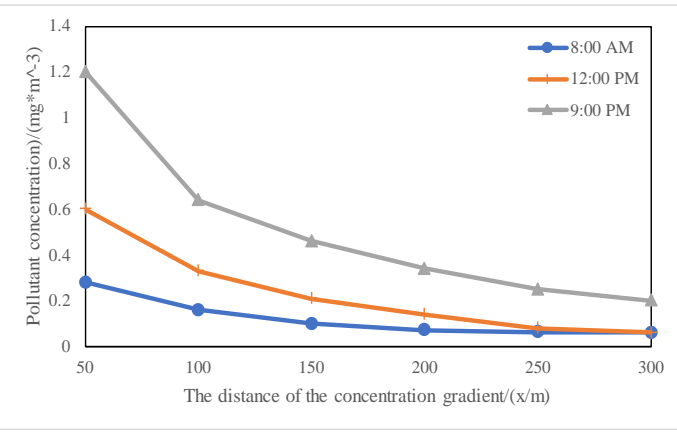

б)

Рис. 3. Изменение кониентрачии загрязнения воздуха в Пекине за три периода:

а) при отсутствии ветра, б) при ветре

Fig. 3. The change in air pollution concentration in Beijing over three periods

На приведенных графиках можно увидеть изменение концентрации загрязнения воздуха на второй, четвертой и шестой кольцевых дорогах Пекина в 8.00, 12.00 и 21.00 при наличии и отсутствии ветра.

\section{Влияние метеорологических факторов окружающей среды}

Степень загрязнения воздуха зависит от процессов конвекции и диффузии. Основными метеорологическими условиями, влияющими на загрязнение воздуха, являются ветер, турбулентность, рельеф местности и слой атмосферного смешивания [5].

Характеристики ветра. Средняя скорость ветра влияет на распространение 3В и определяет степень их разбавления [8]. Чем выше средняя скорость ветра, тем меньше концентрация загрязнения. Направление ветра определяет перемещение загрязнителей. В пограничном слое атмосферы средняя скорость ветра увеличивается с увеличением высоты. В городских районах из-за большого количества зданий скорость ветра на той же высоте ниже, чем в пригороде и сельской местности, меньше и градиент скорости ветра. Следовательно, загрязнения в воздухе над городом быстро перемешиваются, при этом горизонтальная скорость ветра ниже и загрязнение движется медленнее.

Tурбулентность. При распространении турбулентности с увеличением расстояния по ветру периметр дымового облака (факела) постепенно расширяется, а концентрация загрязнения уменьшается (рис. 4).

Чем больше скорость ветра, тем сильнее турбулентность, выше скорость диффузии 3В и ниже концентрация, поэтому ветер и турбулентность являются наиболее важными факторами, определяющими диффузию и разбавление ЗВ в атмосфере.

Слои смешивания. Еще один фактор, который необходимо учитывать при использовании моделей оценки диффузии концентраций 3В, - это отражение слоя смешивания и земли в факеле (облако дыма). «Крыша» и земля слоя смешивания образуют две непроницаемые отражающие стены, в которых ЗВ диффундируют, отражаются и повторно диф-

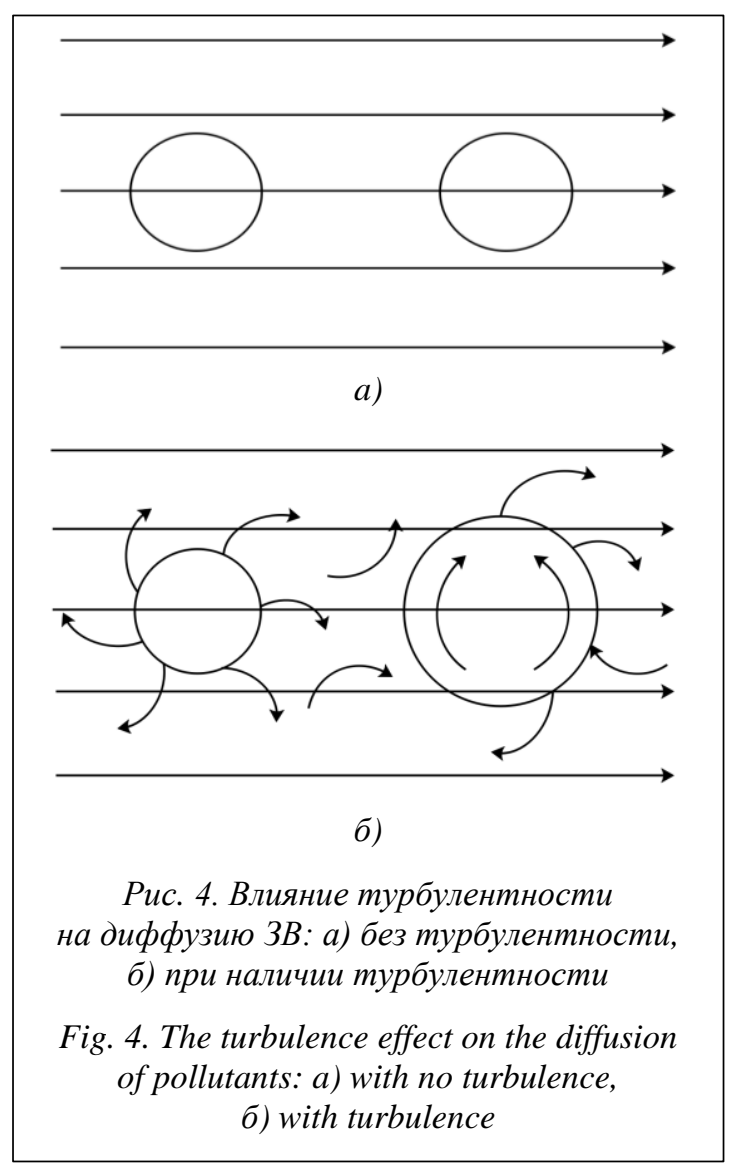


фундируют, что оказывает большое влияние на их распространение.

\section{Математические модели диффузии и конвекции 3В}

Модель диффузии и конвекции точечных источников непрерывных выбросов в атмосферной среде. Основное уравнение диффузии и конвекции $[3,7]$ имеет следующий вид:

$$
\begin{aligned}
& \frac{\partial c}{\partial t}+u_{x} \frac{\partial c}{\partial x}+u_{y} \frac{\partial c}{\partial y}+u_{z} \frac{\partial c}{\partial z}= \\
& =\frac{\partial}{\partial x}\left(D_{x} \frac{\partial c}{\partial x}\right)+\frac{\partial}{\partial y}\left(D_{y} \frac{\partial c}{\partial y}\right)+\frac{\partial}{\partial z}\left(D_{z} \frac{\partial c}{\partial z}\right)+ \\
& +K_{1} X-K_{2} c,
\end{aligned}
$$

где $c$ - концентрация выбросов в окружающей среде; $D_{x}, D_{y}, D_{z}-$ коэффициенты диффузии в направлениях $x, y$ и $z ; u_{x}, u_{y}, u_{z}$ скорости распространения выбросов в направлениях $x, y$ и $z ; K_{1}$ - коэффициент «преобразования» материала источника $x$ в 3В; $K_{2}-$ коэффициент компенсации $3 \mathrm{~B}$ за счет ра-

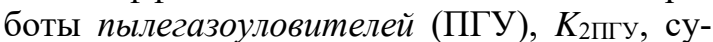
хого и мокрого осадков и химических преобразований, $K_{2 \Sigma} ; K_{2}=K_{2 п г у}+K_{2 \Sigma}$.

Мгновенные значения концентрации загрязнения $n$, выбрасываемые в атмосферу непрерывным точечным источником, вычисляются из распределения в пространстве и времени (нестационарная модель Гаусса) [11]:

$$
\begin{aligned}
& n(x, y, z, t)= \\
& =\frac{Q}{\left(2 \pi^{3 / 2} \sigma_{x} \sigma_{y} \sigma_{z}\right)} \exp \left[-\frac{(x-\bar{u} t)^{2}}{2 \sigma_{x}^{2}}\right] \exp \left[-\frac{y^{2}}{2 \sigma_{y}^{2}}\right] \times \\
& \times \exp \left[-\frac{(z-H)^{2}}{2 \sigma_{z}^{2}}\right]+\exp \left[-\frac{(z+H)^{2}}{2 \sigma_{z}^{2}}\right],
\end{aligned}
$$

где $Q$ - мощность непрерывного источника; $u$ - скорость ветра на высоте $H ; x$ - расстояние от источников; $y$ - поперечное расстояние от оси шлейфа; $z$ - высота над поверхностью земли; $H$ - конечный подъем шлейфа над землей (эффективная высота подъема шлейфа).

Интегрируя по времени концентрацию загрязнений, выбрасываемых из непрерывного источника, можно вычислить установившееся распределение концентрации для стационарной модели Гаусса:

$$
\begin{aligned}
& n(x, y, z, t)=\frac{Q}{\left(2 \pi u \sigma_{x} \sigma_{y} \sigma_{z}\right)} \exp \left[-\frac{y^{2}}{2 \sigma_{y}^{2}}\right] \times \\
& \times\left\{\exp \left[-\frac{(z-H)^{2}}{2 \sigma_{z}^{2}}\right]+\exp \left[-\frac{(z+H)^{2}}{2 \sigma_{z}^{2}}\right]\right\} .
\end{aligned}
$$

В обоих случаях направление ветра совпадает с направлением оси OX, а начало координат лежит в основании источника (трубы). В гауссовой модели также полагается, что имеет место отражение ЗВ от поверхности земли. Отражение характеризуется в формулах членом в фигурных скобках.

Гауссовы модели с различными способами задания $\sigma_{x} \sigma_{y} \sigma_{z}$ имеют свои названия.

Наиболее надежной является модель Пасквилла-Бригса, использование которой официально рекомендуется на расстоянии 100-10 000 метров от источника при шероховатости поверхности $z_{0}=10 \mathrm{~cm}$, но реальное использование этой модели распространяется до нескольких десятков километров. В модели Пасквилла-Бригса значения дисперсий задаются в виде

$$
\sigma_{y}=p_{1} x\left(1+q_{1} x\right)^{-1 / 2}, \sigma_{z}=p_{2} x\left(1+q_{2} x\right)^{-1},
$$

где $p_{i}, q_{i}$ задаются таблично для каждого класса устойчивости атмосферы (класса Пасквилла).

Влияние ветра и отражсение от уровня подстилающей поверхности стационарного ИЗВ. Влияние подстилающей поверхности и «трубы» ИЗВ в точке $P$ рассматривается как сумма двух частей: одна часть - концентрация ЗВ в точке $P$ без учета влияния отражения от подстилающей поверхности, другая - повышенная концентрация ЗВ из-за отражения от нее [11]. Это эквивалентно сумме концентраций 3В в точке $P$, вызванных ИЗВ в точке $(0,0, H e)$ и виртуальным источником в точке $(0,0, \mathrm{He})$ без учета отражения $-c(x, y, z)=c_{1}+c_{2}$. Поэтому значение концентрации [10] можно представить выражением

$$
\begin{aligned}
& c(x, y, z, H e)= \\
& =\frac{Q}{2 \pi u \sigma_{y} \sigma_{z}} e^{-\frac{y^{2}}{2 \sigma_{y}^{2}}}\left(e^{-\frac{(z-H e)^{2}}{2 \sigma_{z}^{2}}}+\alpha e^{-\frac{(z+H e)^{2}}{2 \sigma_{z}^{2}}}\right),
\end{aligned}
$$

где $\alpha$ - коэффициент отражения от подстилающей поверхности.

Поскольку на выбросы, распространяющиеся на большую высоту, будет влиять отражение слоя смешения [12], значение концентрации в любой точке его пространства может быть выражено следующей формулой: $c(x, y, z, \mathrm{He})=$

$$
=\frac{Q}{2 \pi u \sigma_{y} \sigma_{z}} e^{-\frac{y^{2}}{2 \sigma_{y}^{2}}} \sum_{m}\left(e^{-\frac{(z-H e+2 m L)^{2}}{2 \sigma_{z}^{2}}}+\alpha e^{-\frac{(z+H e+2 m L)^{2}}{2 \sigma_{z}^{2}}}\right),
$$


где $L$ - высота слоя смешивания; $m$ - количество отражений (с увеличением $m$ концентрация быстро уменьшается); $H e=H+\Delta H, H-$ высота трубы, $\Delta H$ - высота подъема факела.

\section{Возможные подходы для нейтрализации выбросов}

Рассмотрим концепцию замкнутых систем управления «Природа-Техногеника» (ЗСУПТ) на примере нейтрализации загрязнения при выбросах оксида серы, образующегося при сжигании угля в Пекине [3]. На рисунке 5 представлена функциональная схема мониторинга ЗВ для стационарного ИЗВ, например, теплоэлектроиентраль (ТЭЦ).

Концентрация ЗСУПТ в данном примере иллюстрируется следующей метамоделью.

Будем считать, что $y$ - количество оксида серы, выделяемого после горения угля и выпадающего на приграничной территории; $x-$ количество пара, вырабатываемого ТЭЦ. Получим уравнение баланса: $\frac{d y}{d t}=k_{1} x-k_{2} y$, где $k_{1}$ и $k_{2}$ имеют размерности, обратные времени (сек. $^{-1}, \mathbf{ч}^{-1}$, сут. $^{-1}$ и т.д.); $y$ и $x-$ массы в единицу времени (т/ч, т/сут. и т.д.).
Второй член в приведенном выше уравнении определяет компенсацию за счет химической реакции оксида серы с карбонатом кальция и работы системы очистки.

Учитывая, что за счет обратной связи по комплексному коэффициенту управление положением равно $k_{3} z, z=\gamma y$, можно получить:

$$
\frac{d y}{d t}+\left(k_{2}+k_{3} \gamma\right) y=k_{1} x \text {. }
$$

Предположим, что $x=x_{0}=$ const, тогда уравнение будет следующим:

$$
y=\left(y_{0}-\frac{k_{1}}{k_{2}+k_{3} \gamma} x_{0}\right) e^{-\left(k_{2}+k_{3} \gamma\right) t}+\frac{k_{1}}{k_{2}+k_{3} \gamma} x_{0} .
$$

Очевидно, что ввод управления $k_{3} \gamma$ может радикально снизить выбросы ЗВ. На основе этой концепции, учитывающей уже разработанные общегосударственные мероприятия [3], предложены конструктивные решения по проектированию и производству замкнутых систем минимизации выбросов и стационарных ИЗВ.

Для нейтрализации выбросов от нестационарных ИЗВ (автотранспортных средств) предлагается замкнутая система управления нейтрализацией выбросов автотранспорта

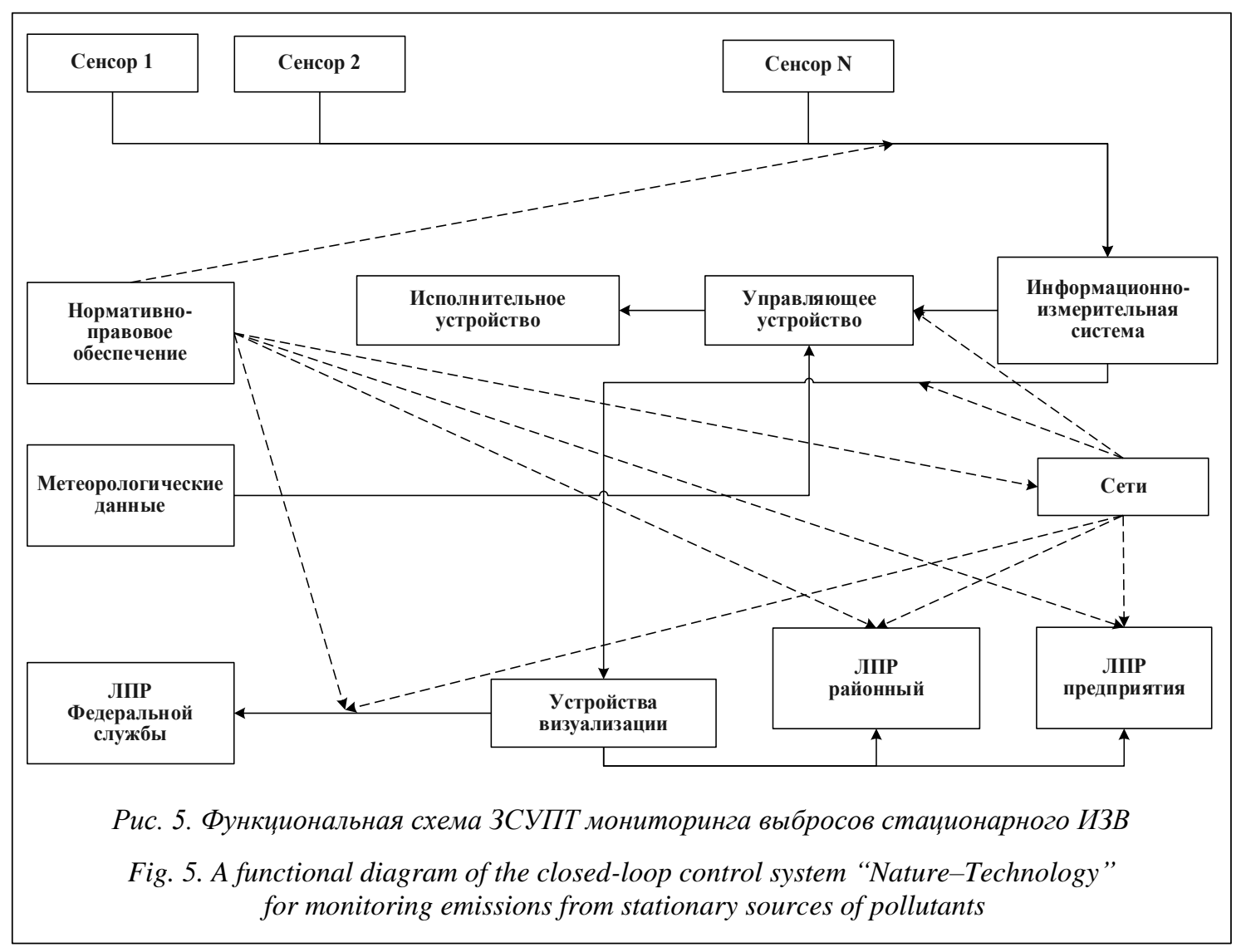


(ЗСУНВА). Покажем концепцию этой системы на примере нейтрализации газа СО выбросов автотранспорта [13]. ЗСУНВА строится аналогично ЗСУНВ стационарных ИЗВ на принципе САУ. Управление осуществляется путем регулирования температуры нагревания катализатора.

На рисунке 6 отражена функциональная схема ЗСУНВА по составляющей выбросов.

Как видим, смесь выхлопных газов поступает на катализатор, в котором происходит химическая реакция нейтрализации. Затем СО проходит через выхлопную трубу к датчику, где измеряется его концентрация. Результат сравнения полученных значений концентраций с ПДК в виде сигнала ошибки поступает на микроконтроллер, генерирующий управляющие сигналы, а затем на исполнительное устройство, в данном случае нагреватель, стабилизирующий температуру катализатора в желаемом диапазоне для наиболее эффективной нейтрализации СО.

\section{Система мониторинга выбросов}

Результаты измерений концентраций 3В в выбросах, как уже отмечалось, подвергаются информационной обработке для дальнейшего применения как в средствах визуализации для ЛПР, так и в ЗСУНВ, ЗСУНВА (рис. 5).

На рисунке 7 показана функциональная схема мониторинга выбросов от ИЗВ.

Система мониторинга характеристик выбросов разработана на основе ПО Protégé и Java [14]. Она может запрашивать характери- стики 3В, входящих в выбросы, и рекомендовать соответствующие защитные меры безопасности населения.

С помощью Protégé можно получить знания (см. http://www.swsys.ru/uploaded/image/ 2021-4/2021-4-dop/8.jpg).

Приобретенные знания и полученные неявные знания отражаются на определенной вкладке Protégé (см. http://www.swsys.ru/uploaded/image/2021-4/2021-4-dop/9.jpg).

Приведем примеры запроса составляющей выбросов на базе языка SPARQL для вызова созданных атрибутов контента [14]:

\{PREFIX pm: <http://www.semanticweb.org/ PotectionMeasures\#>

SELECT ?person?safety_status

WHERE

\{

pm:Naturepm:person ?person.

?personpm:safety_status?safety_status.

\}

\}

Полученный ответ на запрос демонстрируется на экране (см. http://www.swsys.ru/ uploaded/image/2021-4/2021-4-dop/10.jpg).

На основе модулей среды Protégé разработаны средства визуализации [15] выбросов в сети для ЛПР (рис. 5). Эти средства служат также характеристиками ЗВ и для выработки предложений соответствующих защитных мер с точки зрения безопасности. Пользователи осуществляют запросы в следующих форматах [16]:

Варианты 1: Work with Person

Варианты 2: Work with Substance

Варианты 3: Work with Space

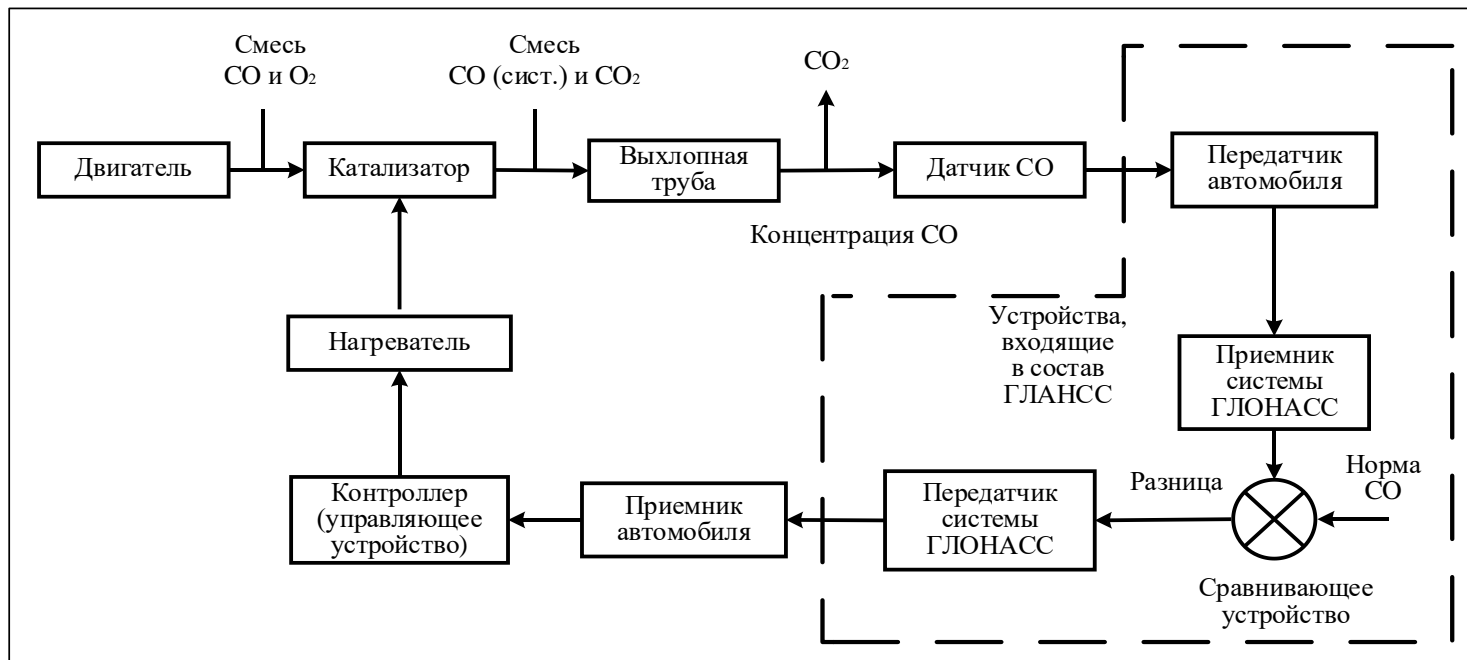

Рис. б. Система нейтрализащчии выхлопных газов автомобилей (СО)

Fig. 6. The Vehicle Exhaust Gas Treatment (CO) 

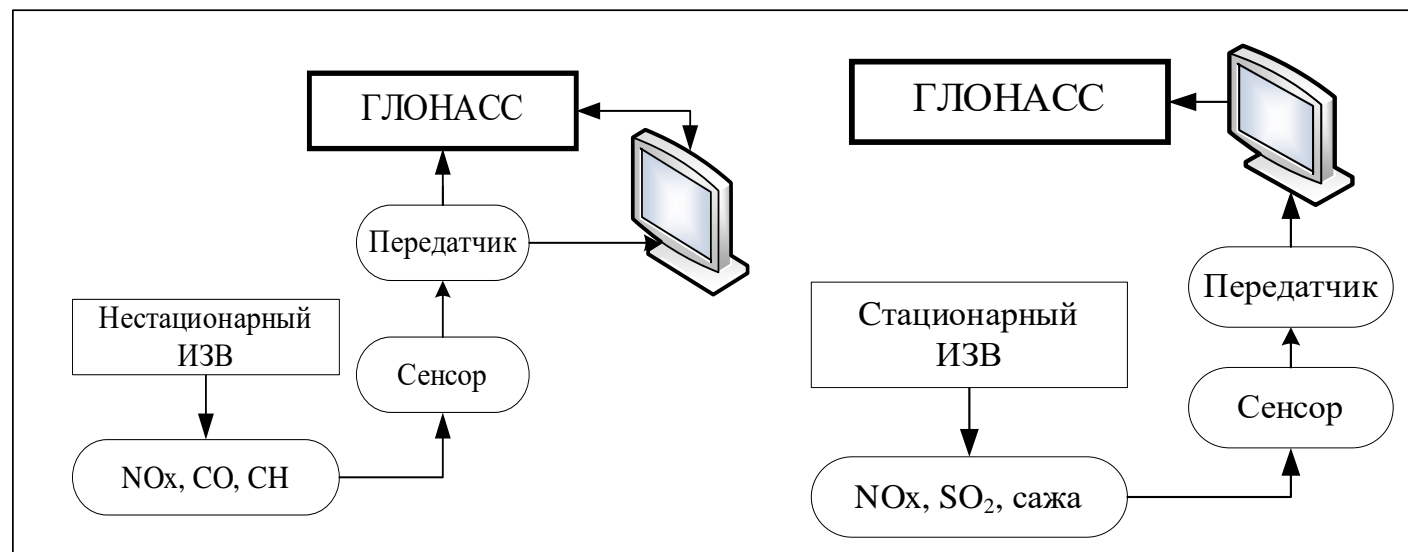

Рис. 7. Схема мониторинга нестационарных и стационарных ИЗВ

Fig. 7. A monitoring scheme for non-stationary and stationary sources of pollutants

Применяются три формы запросов Person, Substance, Space, чтобы обеспечить связанный контент с разных точек зрения.

Примеры интерфейсов каждой формы:

\begin{tabular}{|c|c|c|}
\hline Home & Home & Home \\
\hline Name: Lena & Name: PM 2.5 & Name: Nature \\
\hline Sex: female & Type: particle & Type: outdoor \\
\hline Safety_status: unsafe & & Substances: \\
\hline Age: 41 & & $\mathrm{NO} 2, \mathrm{O} 2, \mathrm{SO} 2$ \\
\hline What to do: wear mask & & Persons: Jane,Tom \\
\hline
\end{tabular}

\section{Применение геоинформационных систем и технологии блокчейн}

Результаты мониторинга загрязнений атмосферы являются пространственно распределенными и привязаны к данным геолокации. Естественно, что их визуализация предполагает использование геоинформационных систем и интерактивных сервисов. В настоящее время известны и доступны для использования в сети Интернет ряд решений, отображающих не только текущую ситуацию, но и данные прогнозирования. Например, на рисунке (см. http://www.swsys.ru/uploaded/ image/2021-4/2021-4-dop/11.jpg) показан прогноз концентрации $\mathrm{NO}_{2}$ в Пекине на интерактивной карте Windy [17] на 28 мая 2021 года к 15:00. Как видно в обозначенной на карте геолокации, она спрогнозирована равной 74,4 мкг/м3. Ряд интерактивных сервисов показывают загрязнение воздуха во многих странах мира [18-20] в виде обобщенных числовых значений индекса качества воздуха AQI (Air Quality Index) [21]. Пример для Пе- кина показан на рисунке (cм. http://www.swsys. ru/uploaded/image/2021-4/2021-4-dop/12.jpg).

Для обмена геоданными разработаны и применяются разнообразные форматы данных, такие как GeoJson [22] (стандарт RFC-7946) на основе JSON и KML [23] на основе XML. Интерактивные карты, доступные в сети Интернет, используют, помимо стандартных, собственные форматы данных для отображения на них маркеров, нагруженных дополнительной информацией.

Измерительные станции фиксируют данные мониторинга состояния воздуха в реальном времени. Во избежание реализации коррупционных сценариев в силу вероятного возникновения потребности представителей загрязняющих предприятий скрыть данные о загрязнениях воздуха очень важно сохранить эти данные таким образом, чтобы они были доступны и в то же время их нельзя было бы изменить. Фиксация данных в обещедоступной информационной среде поможет расследовать экологические преступления.

Для достижения указанных целей пригодна технология распределенного реестра в виде цепочки блоков транзакций (блокчейн) [24]. Надежность хранения данных и их постоянная доступность обеспечиваются многократной непрерывной репликацией БД блокчейна между узлами одноранговой вычислительной сети (blockchain nodes). В развитых сетях блокчейн присутствуют от десятков до тысяч узлов, расположенных в разных юрисдикциях. Защита данных от изменений гарантируется применением односторонних функций (хеш-функций) к данным ранее сохраненных блоков, результаты вычисления 
которых записываются в новые сохраняемые блоки. Права доступа к записи данных реализованы с помощью алгоритмов консенсуса. Для записи экологических геоданных подойдет алгоритм консенсуса из класса Proof Of Stake, не требующий значительных вычислительных ресурсов (например, алгоритм «делегированная византийская отказоустойчивость», используемый в блокчейне NEO [25]). Примером интеграции геоданных в блокчейн является решение, которое записывает в блокчейн данные о геолокации и статусе объекта [26].

Методы, предложенные в данной статье, могут применяться для построения системы мониторинга и принятия решений в области обеспечения технической безопасности, что соответствует целям устойчивого развития.

\section{Заключение}

Авторами данной статьи сделан анализ состояния выбросов в районе Пекина, разработан метод динамически взвешенной оценки, характеристик выбросов, а также построена модель оценки качества воздуха. Кроме того, создана диффузионная модель источников загрязнения атмосферного воздуха. В ходе исследования основного источника загрязнения

- выхлопных газов автомобилей в Пекине были получены градиентное изменение концентрации загрязнения и оценка качества воздуха. Найдено соответствующее решение для нейтрализации загрязнения. На основе ПО Java и Protégé разработана система мониторинга атрибутов загрязнителей воздуха, которая поможет понять, какие загрязнители существуют в различных жизненных средах.

\section{Лumepamypa}

1. Liu J. Research on the temporal and spatial changes of Beijing air pollutants and the evaluation and forecast model. USTB, 2015, pp. 31-62.

2. Wang Z.S. Comparison of Chinese and foreign air quality standards. Environmental Science Research, 2010, no. 3, pp. 253-260.

3. Сольницев Р.И., Коршунов Г.И. Системы управления «Природа-Техногеника». СПб: Политехника, 2012. 205 с.

4. Lu Y.S. Environmental Assessment. China, Tongji University Press Publ., 1999, 695 p.

5. Wu Z.B. Editor-in-chief. Air Pollution Control Technology. China, Science Press, 2002, 520 p.

6. Zhou Z.Y. Impact of meteorological elements on air quality in the Beijing-Tianjin-Hebei region and analysis of future trends. Resource Science, 2014, no. 01, pp. 191-199.

7. Wu Y.M. Modeling the diffusion of a Gaussian plume of atmospheric point source pollution based on GIS. ECNU, 2010, pp. 10-18.

8. Ji Y.K. The establishment of Beijing's air pollutant emission inventory and preliminary research on haze weather. Beijing Jiaotong University, 2015, pp. 3-21.

9. Yin F. Theoretical and experimental studies of dispersion of atmospheric pollutants. Ocean University of China, 2006, pp. 14-25.

10. Сольницев Р.И. Вопросы построения замкнутой системы управления «Природа-Техногеника» // Изв. СПбГЭТУ «ЛЭТИ». 2009. № 7. С. 23-32.

11. Cheng M.Y. Application of Gaussian model in the simulation of multi-point source atmospheric diffusion in small and medium-sized cities. Harbin Normal University, 2020, pp. 20-54.

12. Xu B.L. Parallel method research on Gaussian diffusion model of multi-point source air pollution. J. of Beijing Institute of Technology, 2014, no. 11, pp. 1145-1149.

13. Сольницев Р.И., Коршунов Г.И., Петрушевская А.А., Параничев А.В. Киберфизические системы в экологической безопасности и геомониторинге автотранспорта. СПб: Изд-во СПбГЭТУ «ЛЭТИ», 2019. 147 с.

14. Wang F. Design and implementation of engineering material selection knowledge base based on ontology. Dalian University of Technology, 2016, pp. 15-58.

15. Keet C.M. The African wildlife ontology tutorial ontologies. J. of Biomedical Semantics, 2020, vol. 11, no. 1, pp. 2696-2703. DOI: 10.1186/s13326-020-00224-y.

16. Guan B., Dang D.P. Construction of ontology in the field of financial management. Computer Applications and Software, 2011, vol. 28, no. 02, pp. 10-13.

17. Прогноз концентрации $\mathrm{NO}_{2}$ в Пекине на интерактивной карте Windy на 28 мая 2021 года к 15:00. URL: https://on.windy.com/54ds9 (дата обращения: 10.06.2021).

18. Загрязнение воздуха в Пекине: карта качества воздуха в реальном времени. Снимок. URL: https://aqicn.org/map/beijing/ru/; URL: https://web.archive.org/web/20210528054730/https://aqicn.org/ map/beijing/ru/ (дата обращения: 10.06.2021).

19. World's Air pollution: Real-time AQI. URL: https://waqi.info/ (дата обращения: 10.06.2021). 
20. IQAir Map. Качество воздуха в мире. URL: https://www.iqair.com/ru/air-quality-map (дата обращения: 10.06.2021).

21. Wikipedia. Air Quality Index. URL: https://en.wikipedia.org/wiki/Air_quality_index (дата обращения: 10.06.2021).

22. Butler H., Daly M., Doyle A., Gillies S. et al. The GeoJSON Format, 2018, 28 p. DOI: $10.17487 / \mathrm{rfc} 7946$.

23. OGC. KML. URL: https://www.ogc.org/standards/kml/ (дата обращения: 10.06.2021).

24. Википедия. Блокчейн. URL: https://ru.wikipedia.org/wiki/\%D0\%91\%D0\%BB\%D0\%BE\%D0\% $\mathrm{BA} \% \mathrm{D} 1 \% 87 \% \mathrm{D} 0 \% \mathrm{~B} 5 \% \mathrm{D} 0 \% \mathrm{~B} 9 \% \mathrm{D} 0 \% \mathrm{BD}$ (дата обращения: 10.06.2021).

25. NEO. dBFT 2.0 Algorithm. URL: https://docs.neo.org/v2/docs/en-us/tooldev/consensus/ consensus_algorithm.html (дата обращения: 10.06.2021).

26. Куприянов Г.А., Сольницев Р.И. О программном и информационном обеспечении проектирования и эксплуатации инфраструктуры рециклинга // XXIV Междунар. конф. по мягким вычислениям и измерениям (SCM): сб. докл. 2021. С. 304-307.

Software \& Systems

DOI: $10.15827 / 0236-235 X .136 .649-659$

Received 28.05.21, Revised 23.06.21

2021, vol. 34, no. 4, pp. 649-659

\title{
The environment status analysis and the issues of emission neutralization in China on the example of the Beijing area
}

\author{
R.I. Solnitsev ${ }^{1}$, Dr.Sc.(Engineering), Professor, remira70@mail.ru \\ Lei Wang ${ }^{1}$, Postgraduate Student, 18361206765@163.com \\ S.A. Kuzmin ${ }^{1}$, Ph.D. (Engineering), KSA84@yandex.ru \\ G.A. Kupriyanov ${ }^{1,2}$, Programmer, Research Associate, science@help-in.ru
${ }^{1}$ St. Petersburg Electrotechnical University "LETI", St. Petersburg, 197376, Russian Federation
${ }^{2}$ International Institute of Engineering in Ecology and Human Safety,
St. Petersburg, 197376, Russian Federation

Abstract. This paper is devoted to the problem of compensation for emissions of pollutants into the atmosphere. It shows the application of a comprehensive assessment of the emission concentration in space. An appropriate model of emission propagation is constructed depending on the type of a pollution source. The analysis is based on the data on emissions of pollutants in a specific area of the city of Beijing (China). Based on the analysis of the main stationary and mobile sources (vehicles) of pollution in this area, the authors have determined the gradients of emissions concentration in the atmosphere and the level of air quality during the day.

Based on the analysis results, the paper proposes some approaches and design solutions to compensate for emissions based on the concept of constructing a closed system called Nature-Technology. Such closedloop control systems include means to compensate for emissions from both stationary and non-stationary sources of pollutants. The solution of the control synthesis problems according to the criterion of emission minimization in extreme sections of emission flows for both stationary and non-stationary (motor transport) sources of pollutants is proposed.

The construction of closed-loop emission compensation control systems is associated with forming of an appropriate knowledge base. The knowledge base is based on the ontology components including the types of air pollutants and the ways to compensate for these substances. The ontology contains such concepts as the environment in which a person is currently located, a pollutant that is currently in the environment, and the current degree of compensation for sources of pollutants. In addition to the ontology, the knowledge base contains the rules that make it possible to conclude on the degree of compensation efficiency by the proposed means based on the environmental state including urban infrastructure or an enclosed space, and the type of pollutants (dust, toxic gases, or neutral gases). The ontology was developed in the Protégé editor. The web application for working with the knowledge base is based on JSP technology and the Java programming language. Based on the knowledge base, geographic information systems for data visualization and blockchain technology for registration and reliable storage of data are being built. The paper shows the prospects for the development of this approach for solving the complex problem of compensating emissions from both stationary sources of pollution and from vehicles.

Keywords: emissions, probabilistic characteristic, maximum permissible concentration (MPC), diffusion and convection model, neutralization of pollutants, monitoring system, closed-loop control system, knowledge bases, data storage, blockchain, GIS, geographic information systems. 


\section{References}

1. Liu J. Research on the temporal and spatial changes of Beijing air pollutants and the evaluation and forecast model. USTB, 2015, pp. 31-62.

2. Wang Z.S. Comparison of Chinese and foreign air quality standards. Environmental Science Research, 2010, no. 3, pp. 253-260.

3. Solnitsev R.I., Korshunov G.I. "Nature-Technology" Control Systems. St. Petersburg, 2013, 205 p. (in Russ.)

4. Lu Y.S. Environmental Assessment. China, Tongji University Press Publ., 1999, 695 p.

5. Wu Z.B. Editor-in-Chief. Air Pollution Control Technology. China, Science Press, 2002, 520 p.

6. Zhou Z.Y. Impact of meteorological elements on air quality in the Beijing-Tianjin-Hebei region and analysis of future trends. Resource Science, 2014, no. 01, pp. 191-199.

7. Wu Y.M. Modeling the diffusion of a Gaussian plume of atmospheric point source pollution based on GIS. ECNU, 2010, pp. 10-18.

8. Ji Y.K. The establishment of Beijing's air pollutant emission inventory and preliminary research on haze weather. Beijing Jiaotong University, 2015, pp. 3-21.

9. Yin F. Theoretical and experimental studies of dispersion of atmospheric pollutants. Ocean University of China, 2006, pp. 14-25.

10. Solnitsev R.I. Generating a "Nature-Technogenic" closed control system. Proceedings of Saint Petersburg Electrotechnical University, 2009, no. 7, pp. 23-32 (in Russ.).

11. Cheng M.Y. Application of Gaussian model in the simulation of multi-point source atmospheric diffusion in small and medium-sized cities. Harbin Normal University, 2020, pp. 20-54.

12. Xu B.L. Parallel method research on Gaussian diffusion model of multi-point source air pollution. J. of Beijing Institute of Technology, 2014, no. 11, pp. 1145-1149.

13. Solnitsev R.I., Korshunov G.I., Petrushevskaja A.A., Paranichev A.V. Cyber-physical Systems in Environmental Safety and Geomonitoring of Vehicles. St. Petersburg, 2019, 148 p. (in Russ.).

14. Wang F. Design and implementation of engineering material selection knowledge base based on ontology. Dalian University of Technology, 2016, pp. 15-58.

15. Keet C.M. The African wildlife ontology tutorial ontologies. J. of Biomedical Semantics, 2020, vol. 11, no. 1 , pp. 2696-2703. DOI: 10.1186/s13326-020-00224-y.

16. Guan B., Dang D.P. Construction of ontology in the field of financial management. Computer Applications and Software, 2011, vol. 28, no. 02, pp. 10-13.

17. Forecast of $\mathrm{NO}_{2}$ Concentration in Beijing on the Windy Interactive Map for May 28, 2021 by 15:00. Available at: https://on.windy.com/54ds9 (accessed June 10, 2021) (in Russ.).

18. Beijing Air Pollution: Real-Time Air Quality Map; Snapchot. Available at: https://aqicn.org/map/beijing/; Available at: https://web.archive.org/web/20210528054730/https://aqicn.org/map/beijing/ru/ (accessed June 10, 2021).

19. World's Air Pollution: Real-time AQI. Available at: https://waqi.info/ (accessed June 10, 2021).

20. IQAir Map. World Air Quality. Available at: https://www.iqair.com/ru/air-quality-map (accessed June 10, 2021) (in Russ.).

21. Wikipedia. Air Quality Index. Available at: https://en.wikipedia.org/wiki/Air_quality_index (accessed June 10, 2021).

22. Butler H., Daly M., Doyle A., Gillies S. et al. The GeoJSON Format. 2018, 28 p. DOI: 10.17487/rfc7946.

23. OGC. KML. Available at: https://www.ogc.org/standards/kml/ (accessed June 10, 2021).

24. Wikipedia. Blockchain. Available at: https://en.wikipedia.org/wiki/Blockchain (accessed June 10, 2021).

25. NEO. dBFT 2.0 Algorithm. Available at: https://docs.neo.org/v2/docs/en-us/tooldev/consensus/ consensus_algorithm.html (accessed June 10, 2021).

26. Kupriianov G.A., Solnitsev R.I. On software and information support for the design and operation of the recycling infrastructure. Proc. XXIV Int. Conf. SCM, 2021, pp. 304-307 (in Russ.).

\section{Для цитирования}

Сольницев Р.И., Аэй Ван, Кузьмин С.А., Куприянов Г.А. Анализ состояния окружающей среды и вопросы нейтрализации выбросов в Китае на примере Пекина / / Программные продукты и системы. 2021. Т. 34. № 4. C. 649-659. DOI: 10.15827/0236-235X.136.649-659.

\section{For citation}

Solnitsev R.I., Lei Wang, Kuzmin S.A., Kupriyanov G.A. The environment status analysis and the issues of emission neutralization in China on the example of the Beijing area. Software \& Systems, 2021, vol. 34, no. 4, pp. 649-659 (in Russ.). DOI: 10.15827/0236-235X.136.649-659. 